\title{
Séroprévalence de la toxoplasmose chez les ovins à Bobo-Dioulasso, Burkina Faso
}

\author{
S. Bamba ${ }^{1 *}$ B. Faye ${ }^{2}$ Z. Tarnagda ${ }^{3}$ N. Boly ${ }^{4}$ \\ T. Guiguemdé ${ }^{1}$ I. Villena ${ }^{5}$
}

\begin{abstract}
Mots-clés
Ovin - Toxoplasma gondii Immunodiagnostic - Espace urbain Burkina Faso.
\end{abstract}

\begin{abstract}
Résumé
Une enquête de séroprévalence de la toxoplasmose ovine a été effectuée à Bobo-Dioulasso en 2010. L'objectif de l'étude a été d'évaluer la séroprévalence de la toxoplasmose chez les ovins afin de mieux estimer le risque potentiel que représente leur viande chez les consommateurs. Le test d'agglutination modifié a été utilisé pour le diagnostic sérologique et a révélé une séroprévalence de 58,8 p. 100 (227/386 ; IC à 95 p. 100 : 53,7 - 63,7 p. 100). L'augmentation de la prévalence a été corrélée à l'âge et au sexe (plus importante chez les mâles). Ces résultats indiquent que les toxoplasmes circulent dans le cheptel ovin à Bobo-Dioulasso. L'isolement de T. gondii chez les ovins avec une caractérisation moléculaire des isolats serait nécessaire pour évaluer le risque de la toxoplasmose ovine en santé humaine.
\end{abstract}

1. Laboratoire de parasitologie-mycologie, Institut supérieur des sciences de la santé, Université polytechnique, BP 1091, Bobo-Dioulasso, Burkina Faso.

2. Laboratoire de parasitologie-mycologie, Université Cheikh Anta Diop, Dakar, Sénégal.

3. Institut de recherche en sciences de la santé, Bobo-Dioulasso, Burkina Faso.

4. Abattoir frigorifique, Bobo-Dioulasso, Burkina Faso.

5. Laboratoire de parasitologie-mycologie, Centre national de référence de la toxoplasmose, CHU Maison Blanche, Reims, France.

* Auteur pour la correspondance

Tél. : +22670448075/78 834849

E-mail : hsanata@yahoo.fr les oocystes ou en consommant de la viande peu cuite contenant des kystes tissulaires $(6,10)$. Chez l'homme, la toxoplasmose est en général asymptomatique. Des formes cliniques graves peuvent cependant être observées surtout chez le fotus lors d'une transmission congénitale ou chez des individus immunodéprimés, faisant de cette maladie un problème de santé publique $(3,10)$.

Par ailleurs, la toxoplasmose est une maladie d'intérêt vétérinaire, car elle représente notamment une cause importante d'avortements chez les brebis $(1,6,22)$. Les pertes financières qui en découlent peuvent être potentiellement lourdes pour un pays à vocation agropastorale comme le Burkina Faso (16). De nombreuses enquêtes épidémiologiques désignent la viande comme source importante de contamination humaine $(3,7,10)$. Cependant, il n'y a aucune donnée au Burkina Faso permettant d'estimer ce risque (16). La présente étude se propose d'évaluer la séroprévalence de la toxoplasmose chez les ovins pour une meilleure estimation du risque potentiel que représente la consommation de viande ovine.

\section{MATERIEL ET METHODES}

\section{Contexte et site}

Pays sahélien de $274000 \mathrm{~km}^{2}$, le Burkina Faso est géographiquement enclavé et largement tributaire des aléas climatiques. Son économie repose sur l'agriculture et l'élevage. Ces activités 
occupant plus de 90 p. 100 de la population active contribuent à 40 p. 100 du produit intérieur brut et à 55 p. 100 environ du total des exportations. L'élevage y est de type extensif et constitue la deuxième ressource du secteur primaire du pays. Les ovins sont en majorité destinés à la consommation humaine locale (16).

La ville de Bobo-Dioulasso, située dans la région des Hauts Bassins, au sud-ouest du Burkina Faso, a constitué le site d'étude. Elle est localisée dans la zone Sud soudanienne où la pluviométrie annuelle moyenne est de 1000 à $1300 \mathrm{~mm}$, et la température varie de 16 à $45^{\circ} \mathrm{C}$.

\section{Animaux}

Au total 386 ovins ont été étudiés dont 329 (85,2 p. 100) femelles. Ils provenaient tous de Bobo-Dioulasso, où l'élevage ovin est principalement de type urbain, et ont été sélectionnés de façon aléatoire.

\section{Echantillonnage}

En l'absence de connaissance sur la prévalence de la toxoplasmose chez les ovins au Burkina Faso, la taille de l'échantillon a été calculée avec un logiciel de statistiques (Xlstat) en retenant une précision de 5 p. 100 et une fréquence estimative de 50 p. 100 de séroprévalence de la toxoplasmose chez les ovins à Bobo-Dioulasso (14). Au total, 384 échantillons sanguins de $10 \mathrm{ml}$ chacun ont été prélevés à la veine jugulaire et recueillis dans des tubes stériles. Les échantillons ont été transportés au laboratoire le jour de la collecte, puis centrifugés pendant 15 min à $4000 \mathrm{rpm}$ (Centrifuge $5415 \mathrm{D}$, Germany). Les sérums obtenus ont été conservés à $-20^{\circ} \mathrm{C}$ jusqu'à la réalisation des tests sérologiques.

\section{Test sérologique}

Les échantillons ont été analysés au laboratoire de parasitologie du centre hospitalier et universitaire de Reims, France, par la technique d'agglutination modifiée (MAT). Le principe du test repose sur l'agglutination dans des plaques de microtitration de toxoplasmes formolés (qui constituent l'antigène) par des anticorps (IgG) spécifiques présents dans le sérum des animaux infectés. L'antigène a été préparé selon le protocole de Desmonts et Remington (5). Pour l'analyse, une dilution préalable de chaque sérum au 1/3 dans du tampon phosphate buffer saline (PBS) a été effectuée, puis traitée avec du dithiotréitol (DTT) $(1 \mathrm{M})$ dilué au $1 / 50^{\mathrm{e}}$ dans du tampon PBS pour détruire les IgG. Ensuite, le sérum a été mis en présence des toxoplasmes entiers formolés, dilué au $1 / 17^{\mathrm{e}}$ dans du tampon BABS (Biomérieux). Enfin, une dilution sériée de deux en deux du 1/6 à 1/12 800 a été effectuée. Les plaques contenant tous les sérums traités ont été incubées toute la nuit en dehors de toute source de vibration. Si un sérum contenait des anticorps anti-toxoplasmes, la réaction antigène/anticorps se traduisait par une agglutination des toxoplasmes sous forme d'un voile, indiquant ainsi un résultat positif à la dilution observée. L'absence d'agglutination était synonyme de résultat négatif.

\section{Analyse statistique}

L'analyse des données a été effectuée à l'aide du logiciel Epi-Info version 6.0. Pour l'analyse statistique, le test $\mathrm{du}^{\mathrm{Chi}}{ }^{2}$ a été utilisé (avec $\mathrm{p}<0,05$ considéré comme significatif).

\section{RESULTATS}

Le tableau I montre la répartition des animaux par classes d'âge et ainsi la prédominance des ovins âgés de deux à trois ans
(78,2 p. 100). Au total, 227 ovins $(58,8$ p. 100) possédaient des anticorps anti $T$. gondii. La séroprévalence par tranches d'âge (tableau I) a été la plus élevée chez les animaux âgés de quatre à cinq ans $\left(\mathrm{p}<10^{-3}\right)$. La moyenne d'âge des ovins séropositifs pour $T$. gondii a été de 3,2 ans chez les mâles et de 2,7 ans chez les femelles. La séropositivité a été plus fréquente chez les mâles $(63,2$ p. 100) que chez les femelles $(58,1$ p. 100), mais cette différence n'a pas été significative ( $\mathrm{p}=0,47$, tableau I).

\section{Tableau}

Répartition des ovins en fonction de l'âge et du sexe, et séroprévalence de l'infection à Toxoplasma gondii

\begin{tabular}{|c|c|c|c|c|c|}
\hline & \multicolumn{2}{|c|}{ Ovins testés } & \multicolumn{3}{|c|}{ Séroprévalence } \\
\hline & $\begin{array}{c}\text { Nb. } \\
(\mathrm{n}=386)\end{array}$ & $\%$ & $\begin{array}{c}\text { Nb. } \\
(\mathrm{n}=227)\end{array}$ & $\%$ & IC : 95\% \\
\hline \multicolumn{6}{|c|}{ Age (ans) ${ }^{1}$} \\
\hline $0-2$ & 29 & 7,5 & 10 & 34,5 & $18,6-54,3$ \\
\hline $2-3$ & 302 & 78,2 & 163 & 54 & $48,2-59,7$ \\
\hline $4-5$ & 55 & 14,3 & 54 & 98,2 & $89,0-99,9$ \\
\hline \multicolumn{6}{|l|}{ Sexe $^{2}$} \\
\hline Mâle & 57 & 14,8 & 36 & 63,2 & $49,3-75,2$ \\
\hline Femelle & 329 & 85,2 & 191 & 58,1 & $52,5-63,4$ \\
\hline
\end{tabular}

${ }^{1} \mathrm{p}<0,001 ;{ }^{2} \mathrm{p}=0,47$

\section{DISCUSSION}

La prédominance des ovins femelles observée dans cette étude $(85,2$ p. 100) serait liée aux pratiques religieuses à Bobo-Dioulasso. La majorité des béliers sont conservés dans les élevages pour être vendus, abattus ou sacrifiés en dehors de l'abattoir au cours de cérémonies religieuses musulmanes (fête du mouton) ou d'autres fêtes traditionnelles. Les abattages de routine à l'abattoir concernent ainsi en majorité les femelles. Les animaux de moins de deux ans ont été les moins représentés (7,5 p. 100), ce qui pourrait s'expliquer par le fait que les ovins sont sacrifiés au stade de jeunes adultes (deux à trois ans) ou d'adultes (au delà de trois ans).

La séroprévalence de 58,8 p. 100 relevée dans l'étude a été plus élevée que celle de 43,7 p. $100(\mathrm{n}=300)$ enregistrée en Egypte (19) avec la même technique. Toutefois, des prévalences inférieures ont été relevées chez les ovins au Nigeria (6,7 p. 100 ; 25/372) (12) et en Afrique du Sud (4,3 p. $100 ; 26 / 600)$ (18) avec la technique Elisa. La technique d'agglutination modifiée (MAT) utilisée dans la présente étude est très sensible (seuil de sensibilité de $6 \mathrm{UI} / \mathrm{ml})(5,22)$. Des auteurs ont ainsi rapporté sa bonne sensibilité (90 à 96 p. 100) et sa spécificité $(91,4$ p. 100) chez les ovins et elle peut être utilisée dans les enquêtes épidémiologiques de toxoplasmose ovine $(15,19)$.

La technique Elisa indirect commercialisée est basée sur la mise en évidence d'anticorps dirigés contre la protéine P 30 de Toxoplasma gondii, où toute valeur de la densité optique avec un rapport $\geq 0,5$ (calculée avec un témoin positif) est considérée comme seuil de positivité (22). Par ailleurs, les conditions climatiques ont été associées à la faible prévalence enregistrée au Nigeria. Le même constat a été fait en Afrique du Sud où l'étude avait été conduite dans un climat aride qui ne favorisait pas la survie des oocyste dans le milieu extérieur $(6,11)$. 
Toutefois, malgré la divergence des seuils de sensibilité des techniques MAT et Elisa chez les ovins, des auteurs en France ont noté une bonne concordance (coefficient Kappa $=0,64$ ) entre les deux techniques dans le diagnostic sérologique de la toxoplasmose ovine (22).

Le même constat a été fait en Espagne (15) où des auteurs rapportent une bonne concordance (coefficient Kappa $=0,93$ p. 100) entre les techniques MAT et Elisa chez les ovins. Ailleurs, en Europe, des taux de prévalence également plus faibles de 7,4 p. 100 (109/1 467) ont été notés au Portugal (21) avec la MAT chez les ovins. Les mêmes observations ont été rapportées par des auteurs au Brésil (13) qui ont enregistré chez les ovins un taux de 18,6 p. 100 (71/382) avec la même technique. En Inde (2) et en Iran (17), des auteurs ont relevé avec la même technique des taux de prévalence respectivement de 21,1 p. $100(58 / 276)$ et 44,1 p. 100 (90/204) chez les ovins.

Cependant, des prévalences supérieures aux nôtres de 90 p. 100 (377/419) ont été enregistrées ailleurs en France (22) par la même technique d'agglutination chez les ovins. Ces résultats indiquent que, par la même technique, la prévalence de la toxoplasmose ovine a varié en fonction de la région d'étude.

Par ailleurs, des auteurs rapportent des prévalences supérieures aux nôtres chez les ovins en Grande Bretagne (74 p. 100 ; 2 619/3 539) (9) et en Nouvelle Zélande (85 p. $100 ; 1$ 917/2 254) (4) par le test d'agglutination au latex (technique où l'antigène parasitaire est fixé sur des particules de latex). Au Bengladesh, des auteurs rapportent une prévalence de 40 p. 100 (6/15) par la même technique au latex chez les ovins (20).

La différence de prévalence serait liée à la taille des échantillons étudiés. Les travaux conduits en Grande Bretagne et en Nouvelle Zélande ont en effet utilisé un échantillon important d'ovins (2 200 à 3 500), soit d'une taille de six à neuf fois supérieure à la nôtre. En revanche, au Bengladesh l'étude a concerné 40 ovins, soit 1/26e de l'échantillon de la présente étude. D'autre part, les seuils de sensibilité différents des deux techniques (MAT et agglutination au latex) pourraient justifier cette variabilité du taux de prévalence. Le seuil de sensibilité de la technique d'agglutination au latex a été de titre égal à 1:16 pour les travaux en Grande Bretagne (9), en Nouvelle Zélande (4) et au Bengladesh chez les ovins (20). Des différences géographiques de prévalence ont été observées malgré des effectifs différents, les conditions climatiques constituant un facteur important de la persistance des oocystes dans l'environnement $(7,11)$ après émission de ceux-ci dans les fèces des félins. Ces résultats indiquent que les conditions d'élevage, les facteurs climatiques et environnementaux, notamment la densité des chats, pourraient justifier la variation de la prévalence de la toxoplasmose ovine $(13,17,22)$.

La séroprévalence a augmenté avec l'âge des ovins dans la présente étude. Cette augmentation liée à l'âge a été également rapportée au Nigeria (12), en France $(8,22)$ et en Inde (2). Ceci indique que la contamination est possible à tout âge et que plus une population est exposée aux oocystes de Toxoplasma gondii, plus le risque de contamination est élevé $(7,11)$.

Dans la présente étude, la séroprévalence selon le sexe a montré que les ovins mâles ont été plus fréquemment positifs. Néanmoins, la moyenne d'âge des mâles a été supérieure à celle des femelles et la durée de leur exposition aux oocystes du toxoplasme a ainsi été plus longue $(6,7)$. En outre, les conditions d'hygiène appliquées à l'élevage des ovins influenceraient les niveaux de prévalence. Ainsi, au Burkina Faso et plus précisément dans la ville de BoboDioulasso, les ovins sont élevés dans des conditions d'hygiène et d'alimentation précaires. En effet, les ovins circulent librement dans les concessions, dans les rues, conditions qui les exposent davantage au risque de contamination par les oocystes de toxoplasmes présents dans l'environnement.

\section{- CONCLUSION}

Les résultats de cette étude montrent que la toxoplasmose est présente dans le cheptel ovin de Bobo-Dioulasso. Des conseils d'hygiène et de diététique devraient être diffusés aux consommateurs de viande de mouton et en particulier aux personnes vulnérables (femmes enceintes et patients immunodéprimés non encore immunisés pour cette affection), notamment sur l'importance de la consommation de viande suffisamment cuite.

En outre, étant donné que le Burkina Faso pratique l'élevage des ovins pour la consommation interne et pour l'exportation, l'isolement de $T$. gondii chez les ovins avec une caractérisation moléculaire des isolats sera nécessaire pour mieux évaluer et comprendre le risque de la toxoplasmose ovine sur la santé humaine. Si la présence d'isolats de génotypes virulents était trouvée, cela majorerait le risque d'infestation potentiellement grave pour l'homme.

\section{Remerciements}

Les auteurs remercient le personnel, et en particulier Dr Cathy Chemla, du laboratoire de parasitologie-mycologie, Centre national de référence de toxoplasmose, CHU Maison Blanche, Reims, France. Ils remercient également les bouchers et les techniciens d'élevage de l'abattoir frigorifique de Bobo-Dioulasso, Burkina Faso.

\section{BIBLIOGRAPHIE}

1. ABU-DALBOUH M.A., ABABNEH M.M., GIADINIS N.D., LAFI S.Q., 2012. Ovine and caprine toxoplasmosis (Toxoplasma gondii) in aborted animals in Jordanian goat and sheep flocks. Trop. Anim. Health Prod. 44: 49-54.

2. CHIKWETO A., KUMTHEKAR S., TIWARI K., NYACK B., DEOKAR M.S., STRATTON G., MACPHERSON C.N., SHARMA R.N., DUBEY J.P., 2011. Seroprevalence of Toxoplasma gondii in pigs, sheep, goats, and cattle from Grenada and Carriacou, West Indies. J. Parasitol., 97: 950951.

3. CVETKOVIC D., BOBIC B., JANKOVSKA G., KLUN I., PANOVSKI N., DJURKOVIC-DJAKOVIC O., 2010. Risk factors for Toxoplasma infection in pregnant women in Fyr of Macedonia. Parasite, 17: 183-186.

4. DEMPSTER R.P., WILKINS M., GREEN R.S., DE LISLE G.W., 2011. Serological survey of Toxoplasma gondii and Campylobacter fetus in sheep from New Zealand. N. Z. vet. J., 59: 155-159.

5. DESMONTS G., REMINGTON J.S., 1980. Direct agglutination test for diagnosis of Toxoplasma infection: method for increasing sensitivity and specificity. J. clin. Microbiol, 11: 562-568.

6. DUBEY J.P., 2009. Toxoplasmosis in sheep in the last 20 years. Vet. Parasitol., 163: 1-14.

7. DUBEY J.P., JONES J. L., 2008. Toxoplasma gondii infection in humans and animals in the United States. Int. J. Parasitol., 38: 1257-1278.

8. HALOS L., THEBAULT A., AUBERT D., THOMAS M., PERRET C., GEERS R., ALLIOT A., ESCOTTE-BINET S., AJZENBERG D., DARDE M.L., DURAND B., BOIREAU P., VILLENA I., 2010. An innovative survey underlining the significant level of contamination by Toxoplasma gondii of ovine meat consumed in France. Int. J. Parasitol., 40: 193-200.

9. HUTCHINSON J.P., WEAR A.R., LAMBTON S.L., SMITH R.P., PRITCHARD G.C., 2011. Survey to determine the seroprevalence of Toxoplasma gondii infection in British sheep flocks. Vet. Rec., 169: 582.

10. JONES J.L., KRUEGER A., SCHULKIN J., SCHANTZ P.M., 2010. Toxoplasmosis prevention and testing in pregnancy, survey of obstetrician-gynaecologists. Zoonoses public Health, 57: 27-33. 
11. JONES J.L., DARGELAS V., ROBERTS J., PRESS C., REMINGTON J.S., MONTOYA J.G., 2009. Risk factors for Toxoplasma gondii infection in the United States. Clin. Infect. Dis., 49: 878-884.

12. KAMANI J., MANI A.U., EGWU G.O., 2010. Seroprevalence of Toxoplasma gondii infection in domestic sheep and goats in Borno state, Nigeria. Trop. Anim. Health Prod., 42: 793-797.

13. LANGONI H., GRECA H.J.R, GUIMARAES F.F., ULLMANN L.S., GAIO F.C., UEHARA R.S., ROSA E.P., AMORIM R.M., DA SILVA R.C. 2011. Serological profile of Toxoplasma gondii and Neospora caninum infection in commercial sheep from São Paulo State, Brazil. Vet. Parasitol., 177: 50-54

14. MAGNANI R., 1997. Sampling guide. Impact food security and nutrition monitoring project. Washington DC, USA, Food and Nutrition Technical Assistance, p. 6-8.

15. MAINAR-JAIME R.C., BARBERAN M., 2007. Evaluation of the diagnostic accuracy of the modified agglutination test (MAT) and an indirect ELISA for the detection of serum antibodies against Toxoplasma gondii in sheep through Bayesian approaches. Vet. Parasitol., 148: 122-129.

16. MINISTERE DES RESSOURCES ANIMALES, 2010. Les statistiques du secteur de l'élevage au Burkina Faso. Ouagadougou, Burkina Faso, MRA, direction des Statistiques animales, $171 \mathrm{p}$.

17. RAEGHI S., AKABERI A., SEDEGHI S., 2011. Seroprevalence of Toxoplasma gondii in sheep, cattle and horses in Urmia north-west of Iran. Iran J. Parasitol., 6: 90-94.

\section{Summary}

Bamba S., Faye B., Tarnagda Z., Boly N., Guiguemdé T., Villena I. Seroprevalence of toxoplasmosis in sheep in BoboDioulasso, Burkina Faso

A survey of prevalence of ovine toxoplasmosis was carried out in Bobo-Dioulasso in 2010. The aim of the study was to evaluate the prevalence of toxoplasmosis in sheep in order to better estimate the potential risk of meat for consumers. The modified agglutination test was used for serological diagnosis: the prevalence was 58.8\% (227/386; IC 95\%: 53.7-63.7\%). The increase in prevalence was correlated with age and sex of the animals (higher in males). Results indicate that Toxoplasma circulates in sheep in Bobo-Dioulasso. Isolation of $T$. gondii in sheep with molecular characterization of isolates would be necessary to assess the risk of ovine toxoplasmosis for human health.

Keywords: Sheep - Toxoplasma gondii - Immunodiagnosis Urban space - Burkina Faso.
18. SAMRA N.A., MCCRINDLE CM., PENZHORN B.L., CENCI-GOGA B., 2007. Seroprevalence of toxoplasmosis in sheep in South Africa. J. S. Afr. Vet. Assoc., 78: 116-120.

19. SHAAPAN R.M., EL-NAWAWI F.A., TAWFIK M.A., 2008. Sensitivity and specificity of various serological tests for the detection of Toxoplasma gondii infection in naturally infected sheep. Vet. Parasitol., 153: $359-362$

20. SHAHIDUZZAMAN M., ISLAM R., KHATUN M.M., BATANOVA T.A., KITOH K., TAKASHIMA Y., 2011. Toxoplasma gondii seroprevalence in domestic animals and humans in Mymensingh District, Bangladesh. J. vet. Med. Sci., 73: 1375-1376.

21. SOUSA S., THOMPSON G., SILVA E., FREIRE L., LOPES D., CORREIA DA COSTA J.M., CASTRO A., CARVALHEIRA J., CANADA N., 2009. Determination of the more adequate modified agglutination test cut-off for serodiagnosis of Toxoplasma gondii infection in sheep. Zoonoses public Health, 56: 252-256.

22. VILLENA I, DURAND B., AUBERT D., BLAGA R., GEERS R, THOMAS M., PERRET C., ALLIOT A., ESCOTTE-BINET S., THEBAULT A., BOIREAU P., HALOS L., 2012. New strategy for the survey of Toxoplasma gondii in meat for human consumption. Vet. Parasitol., 183: 203-208.

Accepté le 19.10.2012

\section{Resumen}

Bamba S., Faye B., Tarnagda Z., Boly N., Guiguemdé T., Villena I. Seroprevalencia de la toxoplasmosis en los ovinos de Bobo-Dioulasso, Burkina Faso

Se efectúo una encuesta sobre la seroprevalencia de la toxoplasmosis ovina en Bobo-Dioulasso en 2010. El objetivo del estudio fue el de evaluar la seroprevalencia de la toxoplasmosis en los ovinos, esto con el fin de estimar mejor el riesgo potencial que representa la carne para los consumidores. El test de aglutinación modificada se utilizó para el diagnóstico serológico y reveló una seroprevalencia de 58,8\% (227/386 ; IC a $95 \%$ : 53,7 - 63,7\%). El aumento de la prevalencia estuvo correlacionado con la edad y el sexo (más importante en los machos). Estos resultados indican que los toxoplasmas circulan en el hato ovino en Bobo-Dioulasso. El aislamiento de T. gondii en los ovinos con una caracterización molecular de los aislados sería necesaria para evaluar el riesgo de la toxoplasmosis ovina para la salud humana.

Palabras clave: Ovino - Toxoplasma gondii - Inmunodiagnóstico - Espacio urbano - Burkina Faso. 